\title{
$\beta$-Cyclodextrin Host-Guest Complexes Probed under Thermodynamic Equilibrium: Thermodynamics and AFM Force Spectroscopy
}

Tommaso Auletta, Menno R. de Jong, Alart Mulder, Frank C. J. M. van Veggel, ${ }^{\dagger}$ Jurriaan Huskens, ${ }^{*}$ David N. Reinhoudt*

Supramolecular Chemistry and Technology, MESA ${ }^{+}$Institute for Nanotechnology, University of Twente, P.O. Box 217, 7500 AE Enschede, the Netherlands; Phone: +31-534892980, Fax: +31-534894645, Email:j.huskens@utwente.nl; d.n.reinhoudt@utwente.nl

Shan Zou, Szczepan Zapotoczny, Holger Schönherr, G. Julius Vancso*

Materials Science and Technology of Polymers, MESA ${ }^{+}$Institute for Nanotechnology, University of

Twente, P.O. Box 217, 7500 AE Enschede, the Netherlands; Phone: +31-534892967, Fax: +31-

534893823,E-mail:.g.j.vancso@utwente.nl

Laurens Kuipers

Applied Optics, MESA ${ }^{+}$Institute for Nanotechnology, University of Twente, and FOM Institute for Atomic and Molecular Physics, Kruislaan 407, 1098 SJ Amsterdam, the Netherlands

General Procedures. Melting points were determined with a Reichert melting point apparatus and are uncorrected. NMR spectra were recorded in $\mathrm{CDCl}_{3}$ on a Varian Unity 300 locked to the deuterated solvent at $300.1\left({ }^{1} \mathrm{H}\right), 75.5\left({ }^{13} \mathrm{C}\right)$, and $121.5\left({ }^{31} \mathrm{P}\right) \mathrm{MHz}$, respectively, with tetramethylsilane (TMS) as internal reference. FAB-MS spectra were recorded on a Finnigan MAT 90 spectrometer with $m$ nitrobenzyl alcohol (NBA) as the matrix. Elemental analyses were performed using a Carlo Erba EA1106. Column chromatography was performed using silica gel $\left(\mathrm{SiO}_{2}\right.$, E. Merck, 0.040-0.063 mm, 230-240 mesh).

\footnotetext{
† Present address: University of Victoria, Department of Chemistry, P.O. Box 3065 STN CSC Victoria, BC V8W 3V6, Canada.

\# Present address: Jagiellonian University, Faculty of Chemistry, Ingardena 3, 30-060 Krakow, Poland.
} 
Materials and methods. 2-Mercaptoethanol was purchased from Aldrich and used without any further purification. Synthesis of $\mathbf{1}$ and $\mathbf{4}$ has been reported elsewhere, as well as the preparation and characterization of SAMs of 1. ${ }^{1,2}$ All solvents used in monolayer preparation were of p.a. grade. Compounds 2, 3, 5, and $\mathbf{6}$ were synthesized according to Scheme S1.

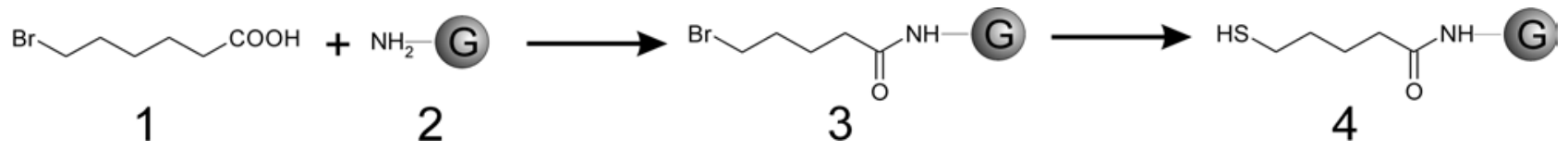

Scheme S1 Synthetic scheme of the thiol modified guests 2, 3, 5, and 6 .

A solution of bromovaleric acid $1(27.6 \mathrm{mmol})$ in oxalyl chloride $(20 \mathrm{~mL})$ was stirred at $40{ }^{\circ} \mathrm{C}$ for $1.5 \mathrm{~h}$. After evaporation of the oxalyl chloride by vacuum distillation, a solution of the amino-guest 2 (27.5 mmol) and triethylamine $(31 \mathrm{mmol})$ in $\mathrm{CH}_{2} \mathrm{Cl}_{2}(20 \mathrm{~mL})$ was added dropwise and the resulting solution was stirred at ambient temperature for $4 \mathrm{~h} . \mathrm{CH}_{2} \mathrm{Cl}_{2}$ was added and the organic layer was washed with $\mathrm{HCl}(1 \mathrm{M})$, water, aqueous $\mathrm{NaOH}(1 \mathrm{M})$, and brine, and dried over $\mathrm{MgSO}_{4}$. Evaporation of the solvent and recrystalization from $\mathrm{HCl}(0.2 \mathrm{M})$ afforded product 3, except for the adamantyl derivative, which was purified by column chromatography.

Potassium carbonate $(3.5 \mathrm{mmol})$ was added to a solution of thioacetic acid $(3.5 \mathrm{mmol})$ in DMF $(20 \mathrm{~mL})$. After dropwise addition of a solution of $3(3.2 \mathrm{mmol})$ in DMF $(10 \mathrm{~mL})$ the solution was stirred at ambient temperature in the dark for $16 \mathrm{~h} . \mathrm{CH}_{2} \mathrm{Cl}_{2}$ and $\mathrm{HCl}(0.5 \mathrm{M})$ were added and the organic layer was washed with $\mathrm{HCl}$ (three times, $0.5 \mathrm{M}$ ), water, and brine. After evaporation of the solvent a degassed 1:2 mixture of aqueous $\mathrm{K}_{2} \mathrm{CO}_{3}(1 \mathrm{M})$ and $\mathrm{MeOH}$ was added and the resulting solution was refluxed for 45 min, poured into $\mathrm{HCl}(1 \mathrm{M})$, and extracted three times with $\mathrm{CH}_{2} \mathrm{CL}_{2}$. The organic layer was washed with $\mathrm{HCl}(1 \mathrm{M})$ and brine, dried $\left(\mathrm{MgSO}_{4}\right)$, and the solvent was evaporated to afford the product 4.

Monolayer preparation and characterization. All glassware used to prepare monolayers was immersed in piraña solution (conc. $\mathrm{H}_{2} \mathrm{SO}_{4}$ and $33 \% \mathrm{H}_{2} \mathrm{O}_{2}$ in a 3:1 ratio). (Warning: piraña should be handled with caution; it has detonated unexpectedly). ${ }^{3}$ The glassware was subsequently rinsed with large amounts of Milli-Q water. Gold substrates were prepared by evaporating $200 \mathrm{~nm}$ of gold on a glass slide of $25 \mathrm{~mm}$ diameter with a $2 \mathrm{~nm}$ titanium layer for adhesion. Just before use, the substrates were cleaned in an oxygen plasma reactor (power: $120 \mathrm{~W}$ ) for $5 \mathrm{~min}$ and the resulting oxide layer was removed by immersing the substrates for $10 \mathrm{~min}$ in $\mathrm{EtOH}^{4}$ Monolayers of compounds 2-6 were prepared by immersing the gold substrates in a $1 \mathrm{mM}$ solution of adsorbates (total concentration of both components) in ethanol at ambient conditions $\left(20 \pm 3^{\circ} \mathrm{C}\right)$ for $16 \mathrm{~h}$. The samples were removed from the 
solution and rinsed with large amounts of dichloromethane, ethanol, and water to remove any physisorbed material. The electrochemical characterization of mixed SAMs of 2-mercaptoethanol and 4 on gold substrates revealed a linear correlation between solution and surface composition at low ferrocene percentage as reported elsewhere. ${ }^{2}$ Contact angle measurements of mixed monolayers of 6 and 2-mercaptoethanol prepared from solutions containing 1\% of adamantylthiol indicate that these are more hydrophobic than monolayers prepared from a solution of 2-mercaptoethanol. Furthermore the advancing contact angles increased with higher percentages of 6 in solution. AFM images of mixed monolayers prepared from solutions containing $20 \%$ and $50 \%$ of 6 did not show any evidence for domain formation of 6 . Mixed SAMs of 2-mercaptoethanol and 6 in different ratios were analyzed by X-ray Photoelectron Spectroscopy (XPS). Linear correlations between the solution composition and the corresponding layers were observed, as reported in Figure S1.

These data indicate that the solution and surface ratios of the adsorbates are equal and no surface phase segregation occurs in the guest concentration range $(0.2-1 \%)$ utilized in the tip functionalization for the force spectroscopy experiments. Such findings were assumed to hold as well for adsorbates $\mathbf{2}, \mathbf{3}$ and $\mathbf{5}$, containing the same alkyl spacer between the guest moiety and the sulfur-anchoring atom.

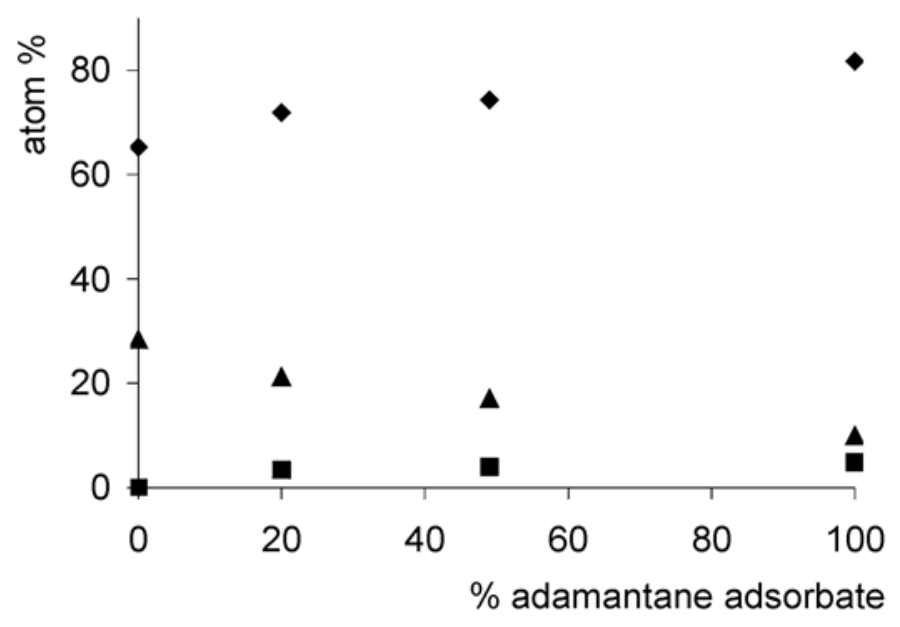

Figure S1. Plot of the content of carbon ( $\bullet$, nitrogen ( $\boldsymbol{\square})$, and oxygen $(\boldsymbol{\Delta})$ of mixed monolayers of 6 and 2-mercaptoethanol as a function of the ratio of 6 to 2-mercaptoethanol in solution, determined by XPS.

Electrochemistry. Electrochemical measurements were performed with an AUTOLAB PGSTAT10, in a custom built electrochemical cell equipped with a platinum counter electrode, a mercury sulfate reference electrode $\left(\mathrm{V}_{\mathrm{MSE}}=+0.61 \mathrm{~V}_{\mathrm{NHE}}\right)$ and a screw cap holding the gold working electrode (area 
exposed to the solution $=0.44 \mathrm{~cm}^{2}$ ). The cyclic voltammograms were performed in $0.1 \mathrm{M} \mathrm{NaClO}_{4}$ at scan rates of $0.1,0.2$, and $0.5 \mathrm{~V} / \mathrm{s}$. The charge values for the electroactive species adsorbed on the electrode and the corresponding standard deviations were calculated by averaging the redox peaks at different scan rates.

Contact Angle Goniometry (CA). The advancing and receding contact angles were measured with Milli-Q water on a Krüss G10 Contact Angle microscope equipped with a CCD camera. Measurements with a drop of water, the volume of which was gradually increased (advancing CA) and then decreased (receding CA), were repeated on three different sites of the sample. For each adsorbate composition three samples were measured for a total of 12 drops, of which the average values and the corresponding errors are reported.

XPS. XPS measurements were performed with a Physical Electronics Quantum2000 equipped with a spherical sector analyzer and a multichannel plate detector (16 detector elements). The analyzer mode was set to constant pass energy. For the survey scan pass energy $=117 \mathrm{eV}$; X-ray beam in high power mode $100 \mathrm{Watt} / 100 \mu \mathrm{m}$; diameter beam scanned over a $1000 \mu \mathrm{m} \times 500 \mu \mathrm{m}$ area; for element scans pass energy $=29.35 \mathrm{eV}$; X-ray beam $25 \mathrm{~W} / 100 \mu \mathrm{m}$; diameter beam scanned over a $1000 \mu \mathrm{m} \times 500 \mu \mathrm{m}$ area. Excitation source: Al K $\alpha$ monochromatic; source energy = $1486.7 \mathrm{eV}$. Take-off angle: 30 degrees (analyzer angle to sample surface). Charge control: both low energy electrons and ions were used to control the surface potential. Temperature during analysis: $298 \mathrm{~K}$. Pressure during analysis: $1-3 \times 10^{-8}$ Torr (argon pressure for charge control). For atomic concentration: Shirley background subtraction. The sensitivity factors were provided by Physical Electronics MultiPack software version 6.1A. The hydrocarbon $\mathrm{C} 1 \mathrm{~s}$ signal at $284.8 \mathrm{eV}$ is used as a reference for surface charging.

MD Simulations. Figure S2 shows the MD simulation data (markers) obtained for a linear dissociation trajectory of ferrocene from a cyclodextrin cavity, according to the procedure described in the Experimental part of the main text. These datapoints were fitted to the LJP function in a leastsquares optimization routine varying $\varepsilon, s$, and $z_{0}$. The resulting optimized fit is shown as the continuous curve in Figure S2. Only the well width $s$ (7.78 $\AA$ ), which correlates well to the cavity depth of cyclodextrin $(8 \AA)$, is used furtheron in the theoretical probability description, as described in the main text. 


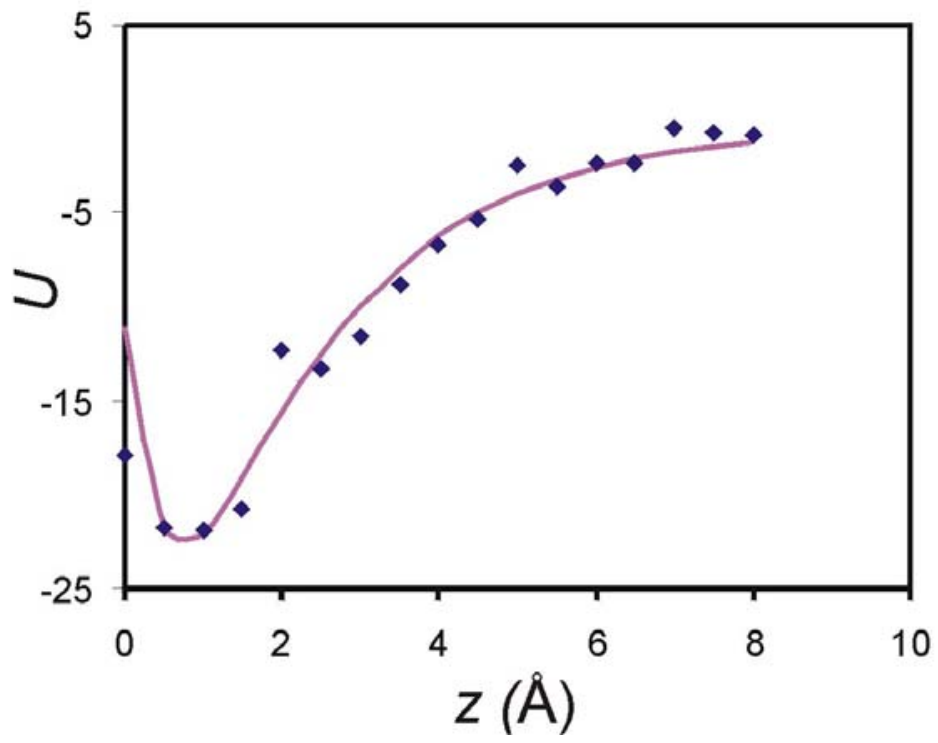

Figure S2. MD simulation data for the interaction energy of the dissociation of ferrocene along a linear dissociation trajectory perpendicular to the center of the cyclodextrin cavity (markers) and the fit to a LJP function (continuous line).

Probability description using approximating harmonic potentials. Alternative to the numerical integration of $p(z)$, using $U_{\text {tot }}(z)$, to give $p_{\text {contact, }}$, as described in the main text, a semi-analytical expression for $p_{\text {contact }}$ can be derived. Here, both the "contact" and "out-of-contact" minima are approximated by a harmonic potential according to equations S1 and S2:

Equation S1 $\quad U_{\text {contact }}=U_{\text {contact } \min }+\frac{1}{2} k_{\text {complex }}\left(z-z_{\text {contact } \text { min }}\right)^{2}$

Equation S2 $\quad U_{\text {out-of-contact }}=U_{\text {out-of-contact, } \text { min }}+\frac{1}{2} k_{\text {tip }}\left(z-z_{\text {out-of-contact, } \text { min }}\right)^{2}$

For well-separated minima, $z_{\text {contact,min }} \approx 0$ and $z_{\text {out-of-contact,min }} \approx z_{1}$, while $U_{\text {contact,min }}$ and $U_{\text {out-of-contact,min }}$ can be approximated by equations S3 and S4.

Equation S3 $\quad U_{\text {contact }} \approx U_{\text {tot }}(0)=-\varepsilon+\frac{1}{2} k_{\text {tip }}\left(z_{1}\right)^{2}$

Equation S4 $\quad U_{\text {out-of-contact }} \approx U_{\text {tot }}\left(z_{1}\right)=4 \varepsilon\left(\left(\frac{s}{z_{1}+z_{0}}\right)^{12}-\left(\frac{s}{z_{1}+z_{0}}\right)^{6}\right)$

To approximate the stiffness of the HG system, a parabola $\left(U=a \times(z-b)^{2}+c\right)$ can be used to fit $U_{t o t}$ in proximity of the "contact" minimum, so that $k_{\text {complex }}=2 a$. The plot of the stiffness of the complex as a function of $\varepsilon$ shows a linear correlation leading to $k_{\text {complex }}=23.81 \varepsilon \mathrm{pN}^{-1}$, with $\varepsilon$ in $\mathrm{kcal} \mathrm{mol}^{-1}$, while $k_{\text {tip }}=8 \mathrm{pN} \AA^{-1}$, as determined for the gold-coated tips experimentally. Since for our systems $\varepsilon$ ranges roughly from 5 to $12 \mathrm{kcal} \mathrm{mol}^{-1}, k_{\text {complex }}$ may range from $125-300 \mathrm{pN} \AA^{-1}$, which is in all cases much higher than the stiffness of the cantilever. It is also seen in the sequence of potential energy graphs 
(Figure 4) and the parabola fittings to the "contact" minima in these graphs (not shown) that a $5 \AA$ linker is already long enough to avoid any linker chain length dependence of the stiffness of the complex.

Now, the probability of being in either minimum, describing the "contact" and the "out-of-contact" states, is governed by the Boltzmann distribution, where each is represented in approximation by its respective harmonic potential. The probability ratio is then given by equation S5.

Equation S5 $\frac{p_{\text {contact }}}{p_{\text {out-of-contact }}}=\frac{\int_{-\infty}^{\infty} e^{-U_{\text {con }}(z) / R T} d z}{\int_{-\infty}^{\infty} e^{-U_{\text {ooc }}(z) / R T} d z}=e^{-\Delta U / R T} \frac{\int_{-\infty}^{\infty} e^{-1 / 2 k_{\text {complex }} z^{2} / R T} d z}{\int_{-\infty}^{\infty} e^{-1 / 2 k_{\text {tip }} z^{2} / R T} d z}=\sqrt{\frac{k_{\text {tip }}}{k_{\text {complex }}}} e^{-\Delta U / R T}$

Here, $\Delta U=U_{\text {contact,min }}-U_{\text {out-of-contact,min }}$. The subsequent transformation into $p_{\text {pull-off }}$ and $F_{\text {pull-off }}$ follows as given in the main text. From this formula, it can be seen that the influence of $k_{\text {complex }}$, which is determined by the well width parameter $s$ and the guest-dependent well depth parameter $\varepsilon$, has only a small influence on $p_{\text {contact }}$, and thus on $p_{\text {pull-off }}$ and $F_{\text {pull-off }}$.

\section{Additional references}

${ }^{1}$ De Jong, M. R.; Huskens, J.; Reinhoudt, D. N. Chem. Eur. J. 2001, 7, 4164-4170.

${ }^{2}$ Auletta, T.; Van Veggel, F. C. J. M.; Reinhoudt, D. N. Langmuir 2002, 18, 1288-1293.

3 a) Dobbs, D. A.; Bergman, R. G.; Theopold, K. H. Chem. Eng. News 1990, 68, (17), 2. b) Wnuk, T. Chem. Eng. News 1990, 68 (26), 2. c) Matlow, S. L. Chem. Eng. News 1990, 68, (30), 2.

${ }^{4}$ Ron, H.; Rubinstein, I. Langmuir 1994, 10, 4566-4573. 